\title{
council of the AMS
}

\section{OFFICERS}

*President: Paul D. Try, Program Director, Science and Technology Corp., 409 Third Street SW, Suite 203, Washington, DC 20024

*President-Elect: Ronald D. McPherson, Director, National Centers for Environmental Prediction, National Weather Service, NOAA, World Weather Building, Rm. 101, 5200 Auth Rd., Washington, DC 20233

*Executive Director: Richard E. Hallgren, American Meteorological Society, 45 Beacon St., Boston, MA 02108

*Secretary-Treasurer: Kenneth C. Spengler, American Meteorological Society, 45 Beacon St., Boston, MA 02108

\section{PAST PRESIDENTS}

*David D. Houghton, Dept. of Atmospheric and Oceanic Sciences, University of Wisconsin-Madison, $1225 \mathrm{~W}$. Dayton St., Madison, WI 53706

*Warren M. Washington, Director, Climate and Global Dynamics Division, National Center for Atmospheric Research, P.O. Box 3000, Boulder, CO 80307-3000

\section{COUNCILORS}

\section{Terms Expire-1997}

Gary M. Carter, Chief, Scientific Services Division, NOAA/ NWS, 630 Johnson Ave., W/ER3, Bohemia, NY 117162618

*Richard S. Greenfield, Director, Division of Atmospheric Sciences, National Science Foundation, 4201 Wilson Blvd., Suite 775, Arlington, VA 22230

Jeremy M. Hales, ENVAIR, 4811 W. 18th Ave., Kennewick, WA 99337

John E. Kutzbach, Center for Climate Research, University of Wisconsin-Madison, 1225 W. Dayton St., Madison, WI 53706-1695
Patricia M. Pauley, Dept. of Meteorology, Naval Postgraduate School, 589 Dyer Rd., Room 254, Monterey, CA 93943 5114

\section{Terms Expire-1998}

William H. Hooke, Director, U.S. Weather Research Program Office/Department of Commerce, NOAA, Office of Oceanic and Atmospheric Research, 1315 East-West Highway, SSMC-3 Room 11360, Silver Spring, MD 20910

Eugenia Kalnay, Chief Development Office, National Centers for Environmental Prediction, World Weather Building, Room 204, W/NMC22, Washington, DC 20233

David A. Randall, Dept. of Atmospheric Science, Colorado State University, Fort Collins, CO 80523

Thomas B. Sanford, Applied Physics Laboratory, University of Washington, 1013 N.E. 40th St., Seattle, WA 98105

Richard C. J. Somerville, Prof. of Meteorology and Director, Climate Research Division, University of California, San Diego, 9500 Gilman Drive, Dept. 0224, La Jolla, CA

\section{Terms Expire-1999}

*Susan K. Avery, Director, CIRES, Campus Box 216, Boulder, CO 80309-3000

Wilfried H. Brutsaert, Cornell University, School of Civil and Environmental Engineering, Hollister Hall, Ithaca, NY 14853-3501

J. Michael Fritsch, Meteorology Dept., The Pennsylvania State University, 503 Walker Building, University Park, PA 16802

Dennis H. McCarthy, National Weather Service, 1200 Westheimer Dr., Rm. 101, Norman, OK 73069-7902

Thomas D. Potter, Director, NWS/WR Headquarters, Box 11188, Federal Building, Rm. 1210, Salt Lake City, UT 84147

*1996 Executive Committee 